\title{
Structural characterization of the $F d d d$ phase in a diblock copolymer thin film by electron microtomography $\dagger$
}

\author{
Jueun Jung, ${ }^{a}$ Hae-Woong Park, ${ }^{a}$ Junyoung Lee, ${ }^{a}$ Haiying Huang, ${ }^{a}$ Taihyun Chang, ${ }^{* a}$ Yecheol Rho, ${ }^{a}$ \\ Moonhor Ree, ${ }^{a}$ Hidekazu Sugimori ${ }^{b}$ and Hiroshi Jinnai ${ }^{c}$
}

Received 1st July 2011, Accepted 18th August 2011

DOI: $10.1039 / \mathrm{c} 1 \mathrm{sm} 06236 \mathrm{k}$

\begin{abstract}
A 3-dimensional $F d d d$ network structure of a polystyrene-block-polyisoprene (PS- $b$-PI) diblock copolymer $\left(M_{\mathrm{n}}=31500, f_{\mathrm{PI}}=0.645\right)$ was observed for the first time in real space by transmission electron microtomography (TEMT). In a $650 \mathrm{~nm}$ thick film of the PS- $b$-PI thin film on a silicon wafer, the $F d d d$ phase was developed after annealing at $215^{\circ} \mathrm{C}$ for $24 \mathrm{~h}$. The single network structure consists of the connected tripodal units of minor PS block domains. The $\{111\}_{\text {Fddd }}$ plane, the densest plane of the minor PS phase, was found to orient parallel to the film plane. The transitional structure from the wetting layer at the free surface to the internal $\{111\}_{\text {Fddd }}$ plane via a perforated layer structure was also observed.
\end{abstract}

\section{Introduction}

Block copolymers exhibit various nano-scale morphologies. In diblock copolymers, lamellar (LAM), hexagonally packed cylinder (HEX), spheres arranged in a body centered cubic lattice (BCC), double gyroid (DG) and hexagonally perforated lamellar (HPL) phases have been well established. ${ }^{1-5}$ Recently another network phase was experimentally found in the bulk state. ${ }^{6-11}$ It has an orthorhombic unit cell and belongs to the Fddd space group $\left(\mathrm{O}^{70}\right)$. The unit cell of the Fddd phase is illustrated in Fig. 1, in which the network skeleton consists of the minor block domain connected with trivalent connectors. ${ }^{6,8}$

Bailey et al. firstly identified the $F d d d$ phase in polyisopreneblock-polystyrene-block-polyethylene oxide (PI- $b$-PS- $b$-PEO) between two-domain lamellar and three-domain lamellar phases and characterized its framework using transmission small angle X-ray scattering (TSAXS) and transmission electron microscopy (TEM). ${ }^{6}$ The Fddd phase has been investigated in diblock copolymers also but to a far lesser extent than triblock copolymers. Kim et al. recently reported on order-order transitions

${ }^{a}$ Department of Chemistry and Division of Advanced Materials Science, Pohang University of Science and Technology (POSTECH), Pohang, 790-784, Korea.E-mail: tc@postech.ac.kr; Fax: +82-54-279-3399; Tel: +82-54-279-2109

${ }^{b}$ Department of Macromolecular Science and Engineering, Graduate School of Science and Engineering, Kyoto Institute of Technology, Kyoto, 606-8585, Japan

'Japan Science and Technology Agency, ERATO, Takahara Soft Interfaces Project and Institute for Materials Chemistry and Engineering (IMCE), CE80, Kyushu University, 744 Motooka, Nishi-ku, Fukuoka, 819-0395, Japan

$\uparrow$ Electronic supplementary information (ESI) available: The experimental procedure of TSAXS and the result for identifying the Fddd phase in the bulk state. Movie showing the 3-D image of the Fddd phase in Fig. 3. See DOI: 10.1039/c1sm06236k from or to the $F d d d$ phase in the polystyrene-block-polyisoprene (PS- $b$-PI) bulk system. ${ }^{12,13}$ In the diblock copolymer, the morphology was observed in a very narrow region between the

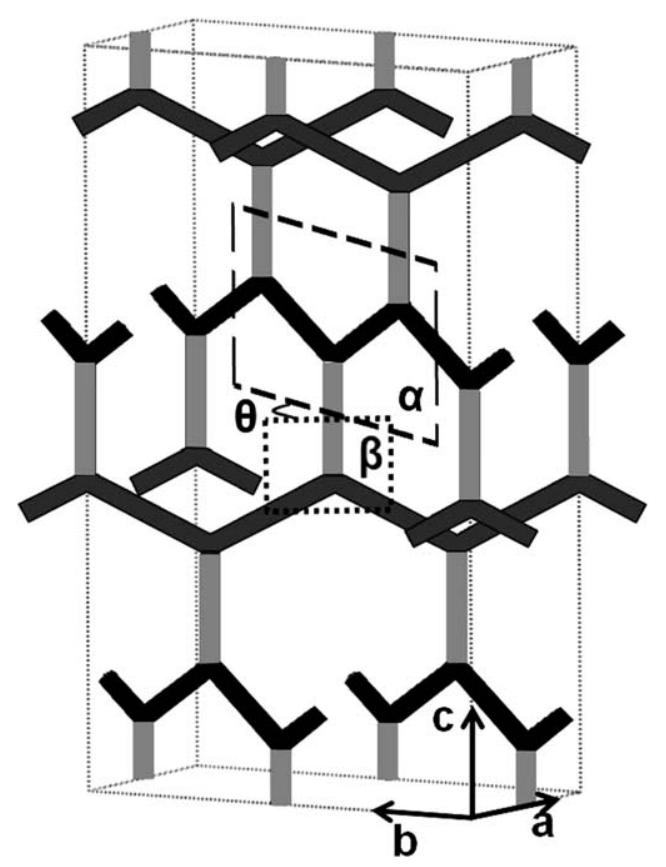

Fig. 1 Schematic representation of a unit cell of the Fddd phase. The solid arrows indicate the direction of each lattice parameter $(a, b$, and $c$ ). In the tripod elementary unit in the $F d d d$ phase, three tripods are on the same plane ( $\alpha$-plane, dashed line rectangle), and the $4^{\text {th }}$ one is twisted ( $\beta$ plane, dotted line rectangle) by an angle, $\theta$. The segments on the $\alpha$-plane, on the $\beta$-plane and parallel to the $c$ axis are color-coded as black, dark gray and gray, respectively. 
LAM and DG boundary as expected from self-consistent field theory and the two-mode expansion method. ${ }^{14-16}$ Since the Fddd phase has a noncubic unit cell and a continuous periodic scaffold, it shows birefringence and highly elastic behavior. ${ }^{6,17}$

Most of the potential applications of block copolymer systems in the nanotechnology field utilize thin film structures. In a thin film, the orientation of block copolymer domains is controlled by chain confinement and preferential wetting. A strong tendency to align the densest plane of the minor phase parallel to the film plane and substantially different phase behaviors relative to the bulk state have been reported. ${ }^{18-25}$ The highly periodic nanopatterns obtained from block copolymers in the film have been exploited for potential applications. ${ }^{26-30} \mathrm{By}$ virtue of the network structure, the $F d d d$ phase is a potential candidate for devices which need large surface channels or a substantial skeleton structure such as membranes or photo-voltaic systems. ${ }^{31-34}$

Although the structure of the $F d d d$ phase has been elucidated based on SAXS and TEM results, ${ }^{6,8}$ 3-dimensional (3-D) observation of the structure has not been reported yet. In this paper, we report on the 3-D observation of the Fddd phase in a diblock copolymer thin film using transmission electron microtomography (TEMT). TEMT allows visualization of a complex morphology in a 3-D real image reconstructed from a series of projective micrographs at different tilting angles of a specimen. ${ }^{35-37}$

\section{Experimental section}

The PS- $b$-PI was prepared by sequential anionic polymerization in cyclohexane at $40{ }^{\circ} \mathrm{C}$ and the detailed synthetic procedure was reported elsewhere. ${ }^{38,39}$ The number-average molecular weight $\left(M_{\mathrm{n}}\right)$ and the polydispersity index $\left(M_{\mathrm{w}} / M_{\mathrm{n}}\right)$ measured by size exclusion chromatography equipped with a light scattering detector (Wyatt, WTREOS-05) were 31500 and 1.02, respectively. The specific refractive index increment $(\mathrm{d} n / \mathrm{d} c)$ was calculated to be $0.145 \mathrm{~mL} \mathrm{~g}^{-1}$ from the block copolymer composition measured by ${ }^{1} \mathrm{H}$ NMR (Bruker, DPX-300) analysis and the $\mathrm{d} n / \mathrm{d} c$ values of PS $\left(0.185 \mathrm{~mL} \mathrm{~g}^{-1}\right)$ and PI $\left(0.120 \mathrm{~mL} \mathrm{~g}^{-1}\right)$ in THF. ${ }^{40}$ The microstructure of the PI block was $74.0 \%$ of 1,4-cis, $20.4 \%$ of 1,4 -trans, and $5.56 \%$ of vinyl. ${ }^{41}$ The volume fraction of PI $\left(f_{\mathrm{PI}}\right)$ was calculated to be 0.645 using the density of PS $\left(1.01 \times 10^{4} \mathrm{~mol} \mathrm{~m}^{-3}\right)$ and PI $\left(1.34 \times 10^{4} \mathrm{~mol} \mathrm{~m}^{-3}\right) .^{42}$

To prepare thin films, the PS- $b$-PI was dissolved in toluene with a small amount $(<1 \mathrm{wt} \%)$ of an antioxidant (Irganox 1010®) to prevent oxidative degradation and crosslinking of the PI block. $10 \mathrm{wt} \%$ polymer solution was spin-coated on a silicon wafer with a $500 \mathrm{~nm}$ thick oxide layer and the thickness of the thin film was measured to be $650 \mathrm{~nm}$ by ellipsometry (J. A. Woollan Co., Inc., EC-400). Before spin coating, the silicon wafer was pre-cleaned with piranha solution and thoroughly rinsed with deionized water. The PS- $b$-PI thin film was annealed above $230{ }^{\circ} \mathrm{C}$ (order-to-disorder transition temperature) for $1 \mathrm{~h}$ under vacuum to erase the preformed structure during the spin coating process, and subsequently annealed at $215^{\circ} \mathrm{C}$ for $24 \mathrm{~h}$ to fully develop the Fddd morphology. The annealed film was quenched rapidly to room temperature $\left(15^{\circ} \mathrm{C} \mathrm{s}^{-1}\right.$ monitored at the sample stage) to preserve the morphology developed at the annealing temperature. ${ }^{23,25}$ The annealing chamber is designed such that air is passed through the sample stage while vacuum is maintained in the system. ${ }^{23,25}$

The specimen for the TEMT experiment was prepared as follows. The annealed film was detached from a silicon wafer by dissolving the oxide layer using aqueous HF solution (3 vol\%) and was picked up with a piece of polystyrene thin plate. A sample was stained with $\mathrm{OsO}_{4}$ (Polyscience, Inc., $0.4 \%$ in water) for $2 \mathrm{~h}$ before embedding with epoxy resin (Polysciences, Inc.). $\mathrm{OsO}_{4}$ stains the PI block selectively in PS- $b$-PI. The epoxyembedded PS- $b$-PI film was microtomed (RMC Ultracut) normal to the film plane at a thickness of $100 \mathrm{~nm}$ and transferred onto a $\mathrm{Cu}$ grid (100 mesh, Ted Pella, Inc.) coated with polyvinyl formal. Gold colloidal particles (GCN005, BB International Co., Ltd., UK) were sprinkled on the sample to serve as fiducial markers for alignment of the projection images. Prior to observation, the TEM specimen was stained once again with $\mathrm{OsO}_{4}$ for 20 min. $^{21,23,25}$

TEMT measurement was performed on an electron microscope (JEM-2200FS, JEOL Co., Ltd., Japan) at an accelerating voltage of $200 \mathrm{kV}$. The instrument was equipped with a $4096 \times$ 4096 slow-scan CCD camera (Gatan USC1000, Gatan Inc., USA) and an energy filter to select transmitted and elastically scattered electrons. The series of the projection images were taken from $-65^{\circ}$ to $+65^{\circ}$ at an angular interval of $1^{\circ}$. The unstained PS domain and fiducial markers are well distinguished from the stained PI block in the projection image obtained even at the highest tilt angle. The image set was aligned by the fiducial marker method. And then the aligned image set was reconstructed by the weighted back projection method which used a weighting filter called a "Shepp-Logan" type filter. ${ }^{35,43}$ The volume fraction of PI determined from the $x-z$ digitally sliced image is 0.628 , which is in reasonable agreement with 0.645 measured by NMR.

\section{Results and discussion}

In bulk, the PS- $b$-PI shows the following phase transition during a continuous temperature scan at $1{ }^{\circ} \mathrm{C} \mathrm{min}-1$ from $120{ }^{\circ} \mathrm{C}$ to $200{ }^{\circ} \mathrm{C}$ (see $\mathrm{ESI} \dagger$ ).

$$
\text { LAM } \underset{136{ }^{\circ} \mathrm{C}}{\longrightarrow} \mathrm{HPL} \underset{150^{\circ} \mathrm{C}}{\longrightarrow} \text { Fddd } \underset{176{ }^{\circ} \mathrm{C}}{\longrightarrow} \mathrm{DG} \underset{192{ }^{\circ} \mathrm{C}}{\longrightarrow} \text { Dis }
$$

The $F d d d$ phase was developed during heating at a temperature range from $150{ }^{\circ} \mathrm{C}$ to $176^{\circ} \mathrm{C}$ between HPL and DG phases. From the peak positions of the TSAXS profile obtained at 160 ${ }^{\circ} \mathrm{C}$, the lattice parameters of the orthorhombic unit cell in the Fddd phase were calculated to be $a: b: c=24.6: 50.0: 89.8 \mathrm{~nm}$. The ratio of the lattice parameters $(0.273: 0.556: 1)$ is quite similar to the one reported by Takenaka et al. for a PS-b-PI Fddd phase in bulk. ${ }^{10}$ Details of the experimental procedure and the TSAXS result for identifying the $F d d d$ phase in the bulk state are elaborated in the ESI $\uparrow$. Looking into the tripodal elementary unit in the $F d d d$ phase illustrated in Fig. 1, three tripods are on the same plane ( $\alpha$-plane), and the $4^{\text {th }}$ one is twisted ( $\beta$-plane) by an angle, $\theta .^{6}$ The angle $\theta$ is calculated to be $52.4^{\circ}$ from the ratio of lattice parameters: $a / b=\tan (\theta / 2)$. If the tripod has a 3-fold symmetry, the lattice parameter of the $F d d d$ phase satisfies the following ratio. ${ }^{6}$ 


$$
a: b: c=\sin (\theta / 2) / \sqrt{ } 3: \cos (\theta / 2) / \sqrt{ } 3: 1
$$

However, the ratio of lattice parameters measured from TSAXS shows a smaller $c$ value, which reflects that the tripodal segments do not have equal lengths and slightly compressed in the $c$-axis of the unit cell. ${ }^{8}$

Thermal phase behavior of block copolymers in the supported thin film is known to be quite different from that of the bulk. ${ }^{11,25,44,45}$ In the previous study with another PS- $b$-PI system $\left(M_{\mathrm{n}}=32.7 \mathrm{k}, f_{\mathrm{PI}}=0.670\right)$, the temperature window of the HPL phase was enlarged while that of DG shrinks as the film gets thinner. ${ }^{25}$ A similar trend of diminishing temperature window in the thin film was also found for both DG and $F d d d$ phases in this study. The Fddd or DG phase was not observed at all during a temperature rising scan at $1{ }^{\circ} \mathrm{C} \min ^{-1}$ in the thin film. It is seemingly due to the retarded kinetics of phase transition in the thin film. ${ }^{25}$ The shrunken temperature window and the retarded phase transition kinetics of the Fddd phase might have not allowed us to observe the phase change via a continuous heating process in situ. Therefore, the phase behavior of the thin film in this study was investigated from individual films annealed at different temperatures for $24 \mathrm{~h}$.

From the individual thin film experiments, the $F d d d$ phase was developed at a temperature range from $215^{\circ} \mathrm{C}$ to $220^{\circ} \mathrm{C}$, which is significantly higher and much narrower than bulk. In addition, the DG phase was not observed in the thin film and the Fddd phase appeared to convert to the DIS (disordered) phase directly upon a further increase in temperature. Although it is hard to conclude whether the DG phase completely disappeared or is hidden from the spot-checking experiments, it is clear that the DG stable temperature range was shrunken greatly in the film. Furthermore, the HPL region is also quite enlarged. The phase behavior of this thin film can be summarized as follows. Since the temperature was not scanned continuously, the phase transition temperatures are reported with some uncertainty. Samples were prepared at every $10{ }^{\circ} \mathrm{C}$ up to $150{ }^{\circ} \mathrm{C}$. From $200{ }^{\circ} \mathrm{C}$ and above, films were prepared at every $5^{\circ} \mathrm{C}$.

$$
\text { LAM } \underset{130{ }^{\circ} \mathrm{C}}{\longrightarrow} \mathrm{HPL} \underset{215^{\circ} \mathrm{C}}{\longrightarrow} F d d d \underset{220{ }^{\circ} \mathrm{C}}{\longrightarrow} \text { Dis }
$$

Fig. 2 shows the cross-sectional TEM image of the PS- $b$-PI thin film after annealing for $24 \mathrm{~h}$ at $215^{\circ} \mathrm{C}$. It shows a wellaligned structure which is analogous to one of the cross-sectional

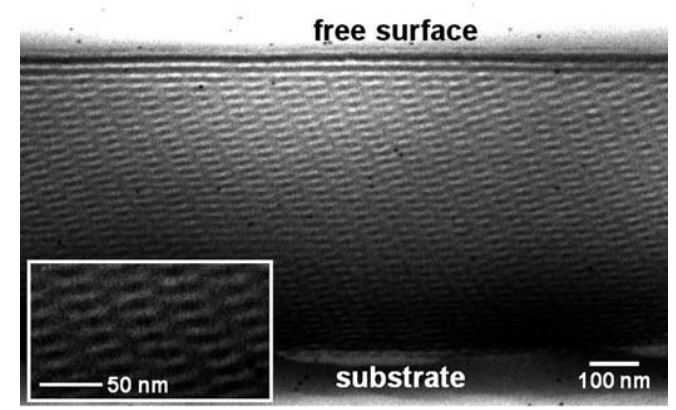

Fig. 2 Cross-sectional TEM micrograph of a PS- $b$-PI thin film annealed at $215^{\circ} \mathrm{C}$ for 1 day. Black dots in the TEM image are due to the spread gold particles as fiducial markers for TEMT experiments. views of the $F d d d$ phase reported earlier. ${ }^{13,46}$ In addition, the layer-like structure developed adjacent to the free surface is also of interest. The TEM image shows a well-developed regular pattern, but it is not sufficient to elucidate the detailed structure from such a projected image. A 3-D TEMT image is much more powerful in this regard.

A reconstructed 3-D image obtained by the TEMT experiment and its digitally sliced images are displayed in Fig. 3. In the reconstructed TEMT image (a), a stained PI domain was made transparent for visual clarity so that the network skeleton of the PS domain can be clearly seen. The PS domain is interconnected by a trivalent junction to make a single network structure unlike DG which consists of two identical networks. ${ }^{23,47,48}$ A 3-D movie of Fig. 3(a) is provided in the ESI $\dagger$. Fig. 3(b) displays three digitally sliced images of the specimen. The position of each cross-sectional plane is indicated as a dotted line ( $x-z$ plane), a dashed line $(y-z$ plane) and a solid line $(x-y$ plane). The $x-y$ plane displays the same plane as the TEM image shown in Fig. 2, but shows higher contrast by virtue of the sliced image. The digitally sliced image of the $y-z$ plane shows a structure consisting of short liner segments at two different orientations. One is parallel to the film plane, which represents the zigzag connection of tripods located at the same plane ( $\alpha$ plane in Fig. 1). The other is tilted at $50-55^{\circ}$ to the film plane, which is derived from the tripods having a different orientation ( $\beta$ plane).
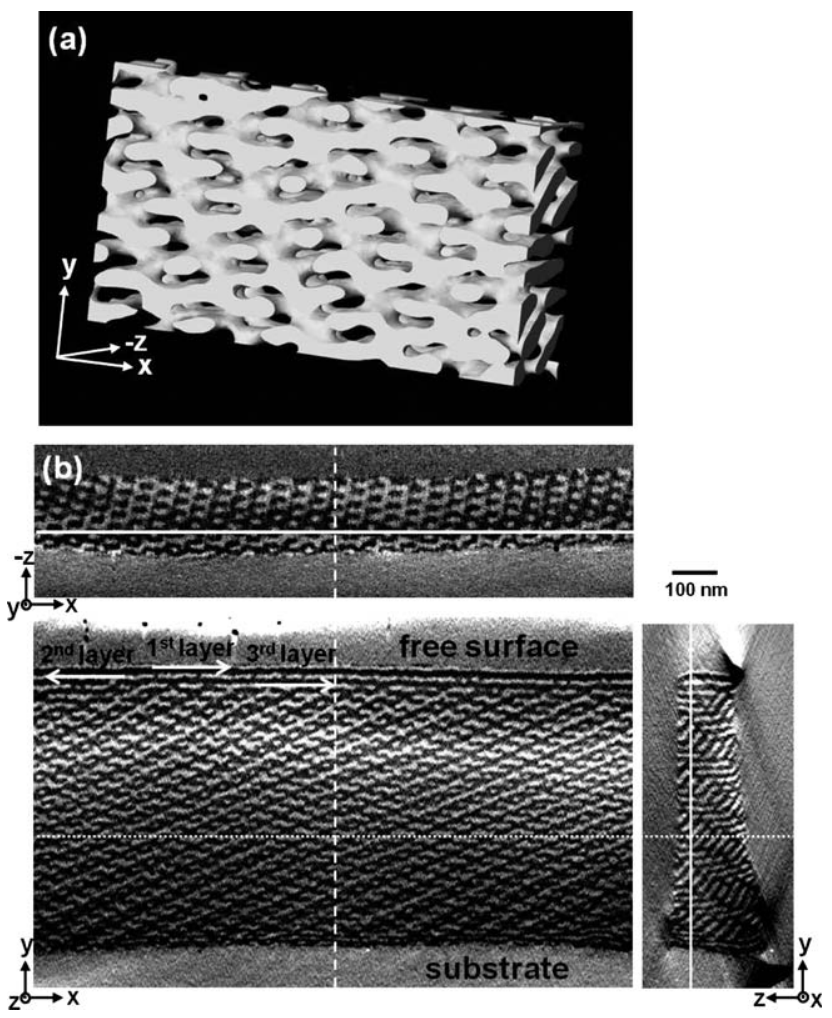

Fig. 3 (a) 3-D reconstructed image of the Fddd phase. Only the PS block domain is shown for visual clarity. The dimension of the 3-D image is $x$ : $y: z=220: 150: 55 \mathrm{~nm}$. (b) Digitally sliced image of each orthogonal plane. Positions of the cross-sectional plane are indicated as a dotted line ( $x-z$ plane), a dashed line ( $y-z$ plane) and a solid line ( $x-y$ plane), respectively. 

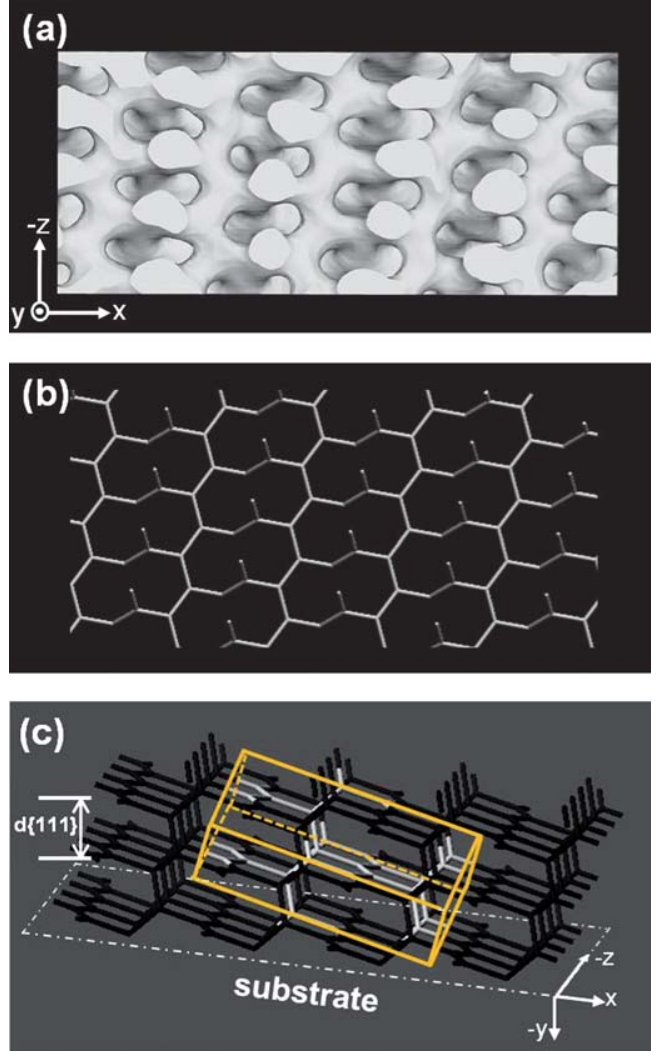

Fig. 4 (a) 3-D reconstructed image $(x: y: z=220: 55: 100 \mathrm{~nm})$ showing the $x-z$ plane which is parallel to the substrate. (b) Model image of the Fddd structure parallel to $\{111\}_{\text {Fddd }}$. This model was constructed using the lattice parameters obtained from the TSAXS result of bulk sample $(a: b: c=24.6: 50.0: 89.8 \mathrm{~nm})$ assuming that all tripodal segments have the same length. (c) The schematic illustration of the unit cell of the Fddd phase in the thin film. The hexahedron is the unit cell and the white skeletons are included in a unit cell. The plane drawn by the dashed-dotted line indicates the substrate (film) plane.

This angle is in accord with the calculated result obtained from the lattice parameter of bulk.

In the thin film, the microdomain of the block copolymer tends to orient spontaneously and the densest plane of the minor component, $\{001\}_{\mathrm{LAM}}, \quad\{003\}_{\mathrm{HPL}}, \quad\{121\}_{\mathrm{DG}}, \quad\{10\}_{\mathrm{HEX}}$ or $\{110\}_{\mathrm{BCC}}$, is aligned parallel to the film plane. ${ }^{19-21,23,25,49,50}$ In Fig. 4, a reconstructed 3-D image showing the $x-z$ plane, parallel to the film plane, is displayed (a) and it is compared with a simulated structure (b). The simulated image is prepared by slicing the modeled Fddd phase parallel to $\{111\}_{\text {Fddd }}$. This Fddd phase model was made using the lattice parameters obtained from the TSAXS result (see ESI $\dagger$ ), and we assumed that all tripodal segments have the same length for simplicity. The structures shown in Fig. 4(a) and (b) are in good agreement. $\{111\}_{\text {Fddd }}$ was shown as the primary peak in the TSAXS profile, and it means this plane is the densest plane of the Fddd phase. In the thin film, $\{111\}_{F d d d}$ is oriented parallel to the film plane as depicted in Fig. 4(c). The unit cell is also drawn in Fig. 4(c). The domain spacing of the $\{111\}_{\text {Fddd }}$ film is calculated from the digitally sliced image to be $20.6 \mathrm{~nm}$, which is in reasonable agreement with $21.4 \mathrm{~nm}$ measured by the TSAXS experiment in bulk. The domain spacing of $\{111\}_{\text {Fddd }}$ is shown in Fig. 4(c). (a) Wetting layer

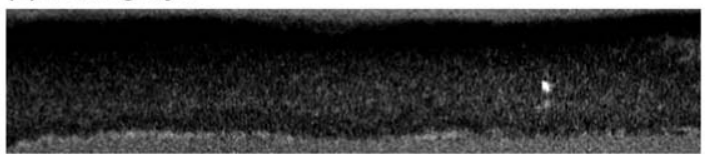

(b) $1^{\text {st }}$ layer

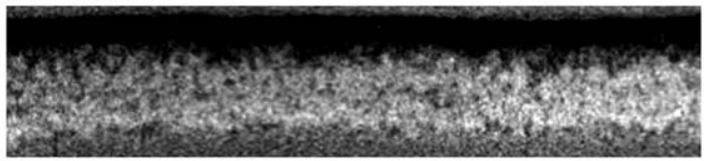

(c) $2^{\text {nd }}$ layer

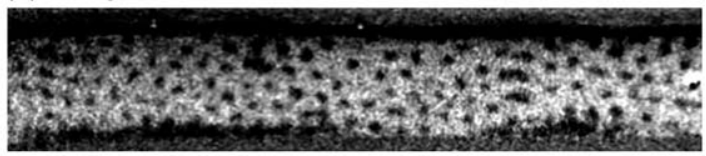

(d) $3^{\text {rd }}$ layer

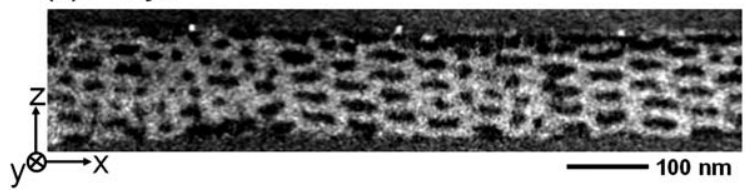

Fig. 5 Digitally sliced images of the $x-z$ plane which is parallel to the free surface. Each layer is marked with arrows in Fig. 3(b). (a) Wetting layer composed of the PI block only, PS layers at different depths: (b) LAM, (c) PL, (d) coexisting phase of PL and Fddd. The $4^{\text {th }}$ layer is similar to Fig. 3(b).

Both Fig. 2 and 3 show a structure of several layers at the free surface which is distinct from the internal network structure. Fig. 5 displays the digitally sliced image of each layer in vicinity of the free surface. The layer in contact with air shown in Fig. 5 (a) is a PI wetting layer because of the low surface energy of the PI block. ${ }^{25,51}$ The PI wetting layer appears dark due to the $\mathrm{OsO}_{4}$ staining. The first PS rich layer next to the PI wetting layer appears as an intact PS layer (b) and it is gradually converted to the Fddd phase. The $2^{\text {nd }}$ PS rich layer (c) is perforated with PI domains and the perforations are not in good order. The next PS rich layer (d) starts to exhibit the characteristic Fddd structure but not perfect yet. The Fddd structure coexists with the perforated layer structure in the third PS rich layer and a perfect Fddd layer like the one shown in Fig. 3(b) is observed from the fourth layer. Such a layer structure at the free surface is persistent after a long-term annealing. A similar transitional layer structure was reported of the DG morphology sample in a thin film, in which the layer structure is converted to the internal DG structure via the HPL structure. ${ }^{25}$ Such deviation of the surface structure from the internal structure occurs due to the preferential wetting of a block and it affects the structure through a few domains near the interface. ${ }^{22,25}$ At the substrate side, on the other hand, no significant structure perturbation appears. It indicates that the silicon oxide surface is not as selective as the free surface in terms of preferential wetting. ${ }^{25}$

\section{Summary}

In summary, we succeeded to develop the $F d d d$ phase of a PS- $b$ PI in a thin film. The $F d d d$ phase was developed over a very narrow temperature range and the OOT of PS- $b$-PI was significantly higher than that in bulk. The 3-D structure of the Fddd 
phase observed by TEMT is consistent with the structure elucidated by the scattering and TEM analysis: a single network structure of tripods. The Fddd phase was well-aligned in the thin film with $\{111\}_{\text {Fddd }}$ parallel to the film plane. The thin films in the Fddd phase were found to exhibit a progressive change from the PI wetting layer, via an intact PS layer and perforated PS layer, to an internal Fddd phase at the free surface. The structural transition takes place over 4-5 layers.

\section{Acknowledgements}

TC acknowledges the supports from NRF via NRL (R0A-2007000-20125-0), SRC (R11-2008-052-03002) and WCU (R31-2008000-10059-0) programs. HJ is grateful for support from the Ministry of Education, Science, Sports and Culture through Grant-in-Aid no. 21015017 and no. 21106512. The SAXS measurements at PAL were supported by the Ministry of Education, Science and Technology and POSCO.

\section{References}

1 L. Leibler, Macromolecules, 1980, 13, 1602-1617.

2 F. S. Bates and G. H. Fredrickson, Annu. Rev. Phys. Chem., 1990, 41, 525-557.

3 M. W. Matsen and F. S. Bates, Macromolecules, 1996, 29, 1091-1098.

4 M. W. Matsen and F. S. Bates, Macromolecules, 1996, 29, 7641-7644.

5 E. W. Cochran, C. J. Garcia-Cervera and G. H. Fredrickson, Macromolecules, 2006, 39, 2449-2451.

6 T. S. Bailey, C. M. Hardy, T. H. Epps and F. S. Bates, Macromolecules, 2002, 35, 7007-7017.

7 E. W. Cochran and F. S. Bates, Phys. Rev. Lett., 2004, 93, 087802.

8 T. H. Epps, E. W. Cochran, T. S. Bailey, R. S. Waletzko, C. M. Hardy and F. S. Bates, Macromolecules, 2004, 37, 8325-8341.

9 T. H. Epps, E. W. Cochran, C. M. Hardy, T. S. Bailey, R. S. Waletzko and F. S. Bates, Macromolecules, 2004, 37, 7085-7088.

10 M. Takenaka, T. Wakada, S. Akasaka, S. Nishitsuji, K. Saijo, H. Shimizu, M. I. Kim and H. Hasegawa, Macromolecules, 2007, 40, 4399-4402.

11 H. Ahn, C. Shin, B. Lee and D. Y. Ryu, Macromolecules, 2010, 43, 1958-1963.

12 M. I. Kim, T. Wakada, S. Akasaka, S. Nishitsuji, K. Saijo, H. Hasegawa, K. Ito and M. Takenaka, Macromolecules, 2008, 41, $7667-7670$.

13 M. I. Kim, T. Wakada, S. Akasaka, S. Nishitsuji, K. Saijo, H. Hasegawa, K. Ito and M. Takenaka, Macromolecules, 2009, 42, 5266-5271.

14 C. A. Tyler and D. C. Morse, Phys. Rev. Lett., 2005, 94, 208302.

15 K. Yamada, M. Nonomura and T. Ohta, J. Phys.: Condens. Matter, 2006, 18, L421-L427.

16 M. W. Matsen, Eur. Phys. J. E, 2009, 30, 361-369.

17 A. J. Meuler, G. Fleury, M. A. Hillmyer and F. S. Bates, Macromolecules, 2008, 41, 5809-5817.

18 A. Knoll, A. Horvat, K. S. Lyakhova, G. Krausch, G. J. A. Sevink, A. V. Zvelindovsky and R. Magerle, Phys. Rev. Lett., 2002, 89, 035501.

19 B. Lee, I. Park, J. Yoon, S. Park, J. Kim, K. W. Kim, T. Chang and M. Ree, Macromolecules, 2005, 38, 4311-4323.

20 G. E. Stein, E. J. Kramer, X. F. Li and J. Wang, Macromolecules, 2007, 40, 2453-2460.
21 H. W. Park, K. Im, B. Chung, M. Ree, T. Chang, K. Sawa and H. Jinnai, Macromolecules, 2007, 40, 2603-2605.

22 X. H. Zhang, B. C. Berry, K. G. Yager, S. Kim, R. L. Jones, S. Satija, D. L. Pickel, J. F. Douglas and A. Karim, ACS Nano, 2008, 2, 23312341.

23 H. W. Park, J. Jung, T. Chang, K. Matsunaga and H. Jinnai, J. Am. Chem. Soc., 2009, 131, 46-47.

24 H. W. Park, J. Jung and T. Chang, Macromol. Res., 2009, 17, 365377.

25 J. Jung, H. W. Park, S. Lee, H. Lee, T. Chang, K. Matsunaga and H. Jinnai, ACS Nano, 2010, 4, 3109-3116.

26 M. J. Fasolka and A. M. Mayes, Annu. Rev. Mater. Res., 2001, 31, 323-355.

27 I. W. Hamley, Soft Matter, 2005, 1, 36-43.

28 I. W. Hamley, Prog. Polym. Sci., 2009, 34, 1161-1210.

29 S. Park, D. H. Lee, J. Xu, B. Kim, S. W. Hong, U. Jeong, T. Xu and T. P. Russell, Science, 2009, 323, 1030-1033.

30 S. H. Park, D. O. Shin, B. H. Kim, D. K. Yoon, K. Kim, S. Y. Lee, S. H. Oh, S. W. Choi, S. C. Jeon and S. O. Kim, Soft Matter, 2010, 6, $120-125$.

31 A. Nykanen, M. Nuopponen, A. Laukkanen, S. P. Hirvonen, M. Rytela, O. Turunen, H. Tenhu, R. Mezzenga, O. Ikkala and J. Ruokolainen, Macromolecules, 2007, 40, 5827-5834.

32 V. N. Urade, T. C. Wei, M. P. Tate, J. D. Kowalski and H. W. Hillhouse, Chem. Mater., 2007, 19, 768-777.

33 E. J. W. Crossland, M. Kamperman, M. Nedelcu, C. Ducati, U. Wiesner, D. M. Smilgies, G. E. S. Toombes, M. A. Hillmyer, S. Ludwigs, U. Steiner and H. J. Snaith, Nano Lett., 2009, 9, 28072812.

34 H. Y. Hsueh, H. Y. Chen, M. S. She, C. K. Chen, R. M. Ho, S. Gwo, H. Hasegawa and E. L. Thomas, Nano Lett., 2010, 10, 49945000 .

35 H. Jinnai and R. J. Spontak, Polymer, 2009, 50, 1067-1087.

36 H. Jinnai, T. Kaneko, K. Matsunaga, C. Abetz and V. Abetz, Soft Matter, 2009, 5, 2042-2046.

37 H. Jinnai, R. J. Spontak and T. Nishi, Macromolecules, 2010, 43, $1675-1688$.

38 K. Kwon, W. Lee, D. Cho and T. Chang, Korea Polym. J., 1999, 7, 321-324.

39 W. Lee, D. Y. Cho, T. Y. Chang, K. J. Hanley and T. P. Lodge, Macromolecules, 2001, 34, 2353-2358.

40 S. Park, D. Cho, J. Ryu, K. Kwon, W. Lee and T. Chang, Macromolecules, 2002, 35, 5974-5979.

41 T. Park, K. Park and T. Chang, Korea Polym. J., 1992, 16, 70 74.

42 S. Park, K. Kwon, D. Cho, B. Lee, M. Ree and T. Chang, Macromolecules, 2003, 36, 4662-4666.

43 H. Sugimori, T. Nishia and H. Jinnai, Macromolecules, 2005, 38, 10226-10233.

44 C. Shin, H. Ahn, E. Kim, D. Y. Ryu, J. Huh, K. W. Kim and T. P. Russell, Macromolecules, 2008, 41, 9140-9145.

45 C. Shin, D. Y. Ryu, J. Huh, J. H. Kim and K. W. Kim, Macromolecules, 2009, 42, 2157-2160.

46 A. J. Meuler, M. A. Hillmyer and F. S. Bates, Macromolecules, 2009, 42, 7221-7250.

47 M. E. Vigild, K. Almdal, K. Mortensen, I. W. Hamley, J. P. A. Fairclough and A. J. Ryan, Macromolecules, 1998, 31, 5702-5716

48 H. Jinnai, Y. Nishikawa, R. J. Spontak, S. D. Smith, D. A. Agard and T. Hashimoto, Phys. Rev. Lett., 2000, 84, 518-521.

49 M. W. Matsen, J. Chem. Phys., 1997, 106, 7781-7791.

50 I. Park, B. Lee, J. Ryu, K. Im, J. Yoon, M. Ree and T. Chang, Macromolecules, 2005, 38, 10532-10536.

51 H. Hasegawa and T. Hashimoto, Macromolecules, 1985, 18, 589590. 